\begin{tabular}{ccc}
\hline & Middle East Journal of Science \\
$\begin{array}{c}\text { INTERNEG } \\
\text { ENGINEERING, } \\
\text { SCIENCE AND } \\
\text { EDUCATION GROUP }\end{array}$ & e-ISSN:2618-6136 & DOI: $10.23884 /$ mejs.2019.5.2.06
\end{tabular}

Research Article

\title{
EXISTENCE RESULTS FOR STEKLOV PROBLEM WITH NONLINEAR BOUNDARY CONDITION
}

\author{
Zehra Yücedă̈ \\ Dicle University, Vocational School of Social Sciences, Turkey \\ *Corresponding author; zyucedag@dicle.edu.tr
}

\begin{abstract}
In this article, we study the nonlinear Steklov boundary value problem. The existence of a nontrivial weak solution is obtained on variable exponent Sobolev spaces, by means of the Mountain Pass theorem.
\end{abstract}

Keywords: $p(x)$-Laplace operator, variational methods, Steklov boundary value, Mountain Pass theorem.

2000 Mathematics Subject Classification: 35J48, 35J60, 35J66.

\begin{tabular}{ll}
\hline Received: November 29, 2019 & Accepted: December 27, 2019 \\
\hline
\end{tabular}

\section{Introduction}

The purpose of this paper is to study the following Steklov problem involving the $\mathrm{p}(\mathrm{x})$-Laplacian,

$$
\left\{\begin{array}{c}
-\operatorname{div}\left(a(x)|\nabla u|^{p(x)-2} \nabla u\right)+|u|^{p(x)-2} u=f(x, u), x \in \Omega \\
a(x)|\nabla u|^{p(x)-2} \frac{\partial u}{\partial v}=|u|^{q(x)-2} u, x \in \partial \Omega
\end{array}\right.
$$

where $\Omega \subset R^{N}(N \geq 2)$ is a bounded with smooth boundary, $p$ is continuous functions on $\bar{\Omega}$ such that $p^{-}:=\inf \underset{x \in \bar{\Omega}}{p}(x)>1, \quad q$ is continuous functions on $\partial \Omega$ such that $q^{-}:=\inf _{x \in \partial \Omega} q(x)>1$, and $p(x) \neq q(y)$ for any $x \in \bar{\Omega}, y \in \partial \Omega, \Delta_{p(x)} u:=\operatorname{div}\left(|\nabla u|^{p(x)-2} \nabla u\right)$ denotes the $p(x)$ - Laplace operator, $f: \Omega x R \rightarrow R$ is a Carathéodory function, $\frac{\partial u}{\partial v}$ is the outer unit normal derivative on $\partial \Omega$ and $a(x)$ is a function which satisfies the conditions $0<a_{1} \leq a(x) \leq a_{2}$ where $a_{1}$ and $a_{2}$ are positive constants.

The study of differential equations and variational problems with $p(x)$ - growth conditions is a new and interesting topic in the last few years. The interest in studying such problems was stimulated by their application in mathematical physics, more precisely in elastic mechanics [25], electrorheological fluids and stationary thermo-rheological viscous flows of non-Newtonian fluids, image processing $[8,12,19,20]$ and the mathematical description of the processes filtration of an idea barotropic gas through a porous medium [3,7]. Many results have been obtained on this kind of problems, for instance, we here cite $[1,4,10,13,14,16,18,21,22]$.

Problems of type $(\mathrm{P})$ has been intensively studied by many authors [2,4,5,6,9,17]. In [24], the authors investigated the existence and multiple results by using a variation of the Mountain Pass the 
following $p(x)$ - Laplacian with nonlinear boundary conditions in bounded domain $\Omega$

$$
\left\{\begin{array}{c}
-\operatorname{div}\left(a(x)|\nabla u|^{p(x)-2} \nabla u\right)+b(x)|u|^{p(x)-2} u=\lambda f(x, u), x \in \Omega \\
a(x)|\nabla u|^{p(x)-2} \frac{\partial u}{\partial v}=c(x)|u|^{q(x)-2} u+\eta g(x, u), x \in \partial \Omega
\end{array}\right.
$$

where $f$ and $g$ functions satisfies the Ambrosetti-Rabinowitz type condition. We also mention that the authors [11] studied de existence result for following class of Steklov boundary value problems involving $p(x)$ - Laplacian

$$
\left\{\begin{array}{c}
-\Delta_{p(x)} u+a(x)|u|^{p(x)-2} u=f(x, u), x \in \Omega \\
|\nabla u|^{p(x)-2} \frac{\partial u}{\partial v}=g(x, u), x \in \partial \Omega
\end{array}\right.
$$

Using the variational method, under suitable conditions $a, f$ and $g$, they obtained results on the existence of solutions.

This paper is organized as follows. In Section 2, we present some necessary preliminary knowledge on variable exponent Lebesgue and Sobolev spaces. In Section 3, using Mountain Pass theorem and the variational method we show the existence nontrivial weak solutions of problem $(\mathrm{P})$.

\section{Preliminaries}

In this section, we recall in what follows some definitions and basic properties of variable exponent Lebesgue-Sobolev spaces $L^{p(x)}(\Omega), W^{1, p(x)}(\Omega)$ and $W_{0}^{1, p(x)}(\Omega)[22,16,15]$.

Set $\mathrm{C}_{+}(\bar{\Omega})=\left\{\mathrm{p} ; \mathrm{p}(\mathrm{x}) \in \mathrm{C}_{+}(\bar{\Omega})\right.$, inf $\mathrm{p}(\mathrm{x})>1$, for all $\left.\mathrm{x} \in \bar{\Omega}\right\}$.

For any $\mathrm{p}(\mathrm{x}) \in \mathrm{C}_{+}(\bar{\Omega})$, we denote

$$
1<p^{-}:=\inf _{x \in \Omega} p(x) \leq p^{+}:=\sup _{x \in \Omega} p(x)<\infty .
$$

Define the variable exponent Lebesgue space $L^{p(x)}(\Omega)$ by

$$
L^{p(x)}(\Omega)=\left\{u \mid u: \Omega \rightarrow R \text { is measurable, such that } \int_{\Omega}|\mathrm{u}(\mathrm{x})|^{p(x)} d x<\infty\right\}
$$

We define a norm, the so-called Luxemburg norm, on this space by the formula

$$
|u|_{p(x)}:=\inf \left\{\lambda>0: \int_{\Omega}\left|\frac{\mathrm{u}(\mathrm{x})}{\lambda}\right|^{p(x)} d x \leq 1\right\}
$$

and $\left(L^{p(x)}(\Omega),|u|_{p(x)}\right)$ becomes a Banach space.

Let $a: \partial \Omega \rightarrow R$ be measurable. Define the weighted variable exponent Lebesgue space

$$
L_{a(x)}^{p(x)}(\partial \Omega)=\left\{u \mid u: \partial \Omega \rightarrow R \text { is measurable and } \int_{\partial \Omega}|a(x)||u|^{p(x)} d x<+\infty\right\},
$$

With the norm

$$
|u|_{(p(x), a(x))}=\inf \left\{\kappa>0 ;\left.\int_{\partial \Omega}|a(x)| \frac{u}{\kappa}\right|^{p(x)} d \sigma \leq 1\right\}
$$


Where $d \sigma$ is the measure on the boundary. Then $L_{a(x)}^{p(x)}(\partial \Omega)$ is a Banach space. In particular, $a \in L^{\infty}(\partial \Omega), L_{a(x)}^{p(x)}(\partial \Omega)=L^{p(x)}(\partial \Omega)$.

Proposition 2.1[14,21] If $p(x) \in L^{\infty}$, the conjugate space $L^{p(x)}(\Omega)$ is $L^{p^{\prime}(x)}(\Omega)$ where $\frac{1}{p(x)}+\frac{1}{p^{\prime}(x)}=1$. For any $u \in L^{p(x)}(\Omega)$ and $v \in L^{p^{\prime}(x)}(\Omega)$, we have

$$
\left|\int_{\Omega} u v d x\right| \leq\left(\frac{1}{p^{-}}+\frac{1}{p^{+}}\right)|u|_{p(x)}|v|_{p^{\prime}(x)} .
$$

The modular of the $L^{p(x)}(\Omega)$ space, which is the mapping $\rho_{p(x)}: L^{p(x)}(\Omega) \rightarrow R$ defined by

$$
\rho_{p(x)}(u)=\int_{\Omega}|u|^{p(x)} d x, \quad \forall u \in L^{p(x)}(\Omega) .
$$

Proposition 2.2 [15,24] If $u, u_{n} \in L^{p(x)}(\Omega)(n=1,2, \ldots)$ and $p^{+}<\infty$, we have

$$
\begin{aligned}
& |u|_{p(x)}<1(=1,>1) \Leftrightarrow \rho_{p(x)}(u)<1(=1,>1), \\
& \min \left(|u|_{p(x)}^{p^{-}},|u|_{p(x)}^{p^{+}}\right) \leq \rho_{p(x)}(u) \leq \max \left(|u|_{p(x)}^{p^{-}},|u|_{p(x)}^{p^{+}}\right), \\
& \left|u_{n}-u\right|_{p(x)} \rightarrow 0(\rightarrow \infty) \Leftrightarrow \rho_{(p(x), \Omega)}\left(u_{n}-u\right) \rightarrow 0(\rightarrow \infty) .
\end{aligned}
$$

Proposition 2.3 [24] Denote $\rho_{p(x)}(u)=\int_{\partial \Omega}|u|^{p(x)} d \sigma, \forall u \in L^{p(x)}(\partial \Omega)$. Then

$$
\begin{gathered}
|u|_{L^{p(x)}(\partial \Omega)} \geq 1 \Leftrightarrow|u|_{L^{p(x)}(\partial \Omega)}^{p^{-}} \leq \rho_{L^{p(x)}(\partial \Omega)}(u) \leq|u|_{L^{p(x)}(\partial \Omega)}^{p^{+}}, \\
|u|_{L^{p(x)}(\partial \Omega)}<1 \Leftrightarrow|u|_{L^{p(x)}(\partial \Omega)}^{p^{+}} \leq \rho_{L^{p(x)}(\partial \Omega)}(u) \leq|u|_{L^{p(x)}(\partial \Omega)}^{p^{-}} .
\end{gathered}
$$

Remark 2.4. It is noted that since $L^{p(x)}(\Omega) \rightarrow L_{l o c}^{1}(\Omega)$ i.e., for any compact subset $K \subset \Omega$ there exists a constant $C_{K}>0$ such that $\left\|f X_{K}\right\|_{1} \leq C_{K}\|f\|$. So every function in $L^{p(x)}(\Omega)$ has a distributional (weak) derivative, and variable exponent Sobolev space is well defined on $L^{p(x)}(\Omega)$.

The variable exponent Sobolev space $W^{1, p(x)}(\Omega)$ is denined by

$$
W^{1, p(x)}(\Omega)=\left\{u \in L^{p(x)}(\Omega):|\nabla u| \in L^{p(x)}(\Omega)\right\}
$$

and equipped with the norm,

$$
\|u\|:=\inf \left\{\lambda>0: \int_{\Omega}\left|\frac{\nabla u(\mathrm{x})}{\lambda}\right|^{p(x)}+\int_{\Omega}\left|\frac{\mathrm{u}(\mathrm{x})}{\lambda}\right|^{p(x)} d x \leq 1\right\} .
$$

For $u \in W^{1, p(x)}(\Omega)$, if we define

$$
\|u\|_{a}:=\inf \left\{\lambda>0: \int_{\Omega} a(x)\left|\frac{\nabla u(\mathrm{x})}{\lambda}\right|^{p(x)}+\int_{\Omega} b(x)\left|\frac{\mathrm{u}(\mathrm{x})}{\lambda}\right|^{p(x)} d x \leq 1\right\} .
$$

In view of assumptions $a(x)$ of and $b(x)(b(x)$ is a function which satisfies the conditions $0<b_{1} \leq b(x) \leq b_{2}$ where $b_{1}$ and $b_{2}$ are positive constants ), it is easy to see that $\|u\|_{a}$ is an equivalent norm on $W^{1, p(x)}(\Omega)$.

Proposition $2.5[24]$ Denote $\Gamma(u)=\int_{\partial \Omega}\left(a(x)|\nabla u|^{p(x)}+|u|^{p(x)}\right) d \sigma, \forall u \in W^{1, p(x)}(\partial \Omega)$. Then 
(i) $\quad \Gamma(u) \geq 1 \Rightarrow \varepsilon_{1}\|u\|^{p^{-}} \leq \Gamma(u) \leq \varepsilon_{2}\|u\|^{p^{+}}$

(ii) $\quad \Gamma(u) \leq 1 \Rightarrow \varepsilon_{3}\|u\|^{p^{+}} \leq \Gamma(u) \leq \varepsilon_{4}\|u\|^{p^{-}}$

where $\varepsilon_{1}, \varepsilon_{2}, \varepsilon_{3}$ and $\varepsilon_{4}$ are positive constants independent of $u$.

Space $W_{0}^{1, p(x)}(\Omega)$ is denoted as the closure of $C_{0}^{\infty}(\Omega)$ in $W^{1, p(x)}(\Omega)$ with respect to the norm $|u|_{1, p(x)}$. For $u \in W_{0}^{1, p(x)}(\Omega)$, we can define an equivalent norm $\|u\|=|\nabla u|_{p(x)}$. Since Poincaré inequality holds [16], i.e. there exists a positive constant $C>0$ such that

$$
\|u\| \leq C|\nabla u|_{p(x)} \text { for all } u \in W_{0}^{1, p(x)}(\Omega) .
$$

\section{Proposition 2.6 [16,24]}

(i) If $1<p^{-} \leq p^{+}<\infty$ then the spaces $L^{p(x)}(\Omega), W^{1, p(x)}(\Omega)$ and $W_{0}^{1, p(x)}(\Omega)$ are separable, reflexive and uniformly convex Banach spaces,

(ii) If $\mathrm{q}(\mathrm{x}) \in \mathrm{C}_{+}(\bar{\Omega})$ and $\mathrm{q}(\mathrm{x})<p^{*}(x)$ for all $\mathrm{x} \in \bar{\Omega}$ then the embedding $W^{1, p(x)}(\Omega) \rightarrow L^{q(x)}(\Omega)$ is compact and continuous,

(iii) If $\mathrm{q}(\mathrm{x}) \in \mathrm{C}_{+}(\partial \Omega) \mathrm{q}(\mathrm{x})<p^{\partial}(x)$ and for all $\mathrm{x} \in \partial \Omega$ then the trace embedding $W^{1, p(x)}(\Omega) \rightarrow L^{q(x)}(\partial \Omega)$ is compact and continuous.

We define,

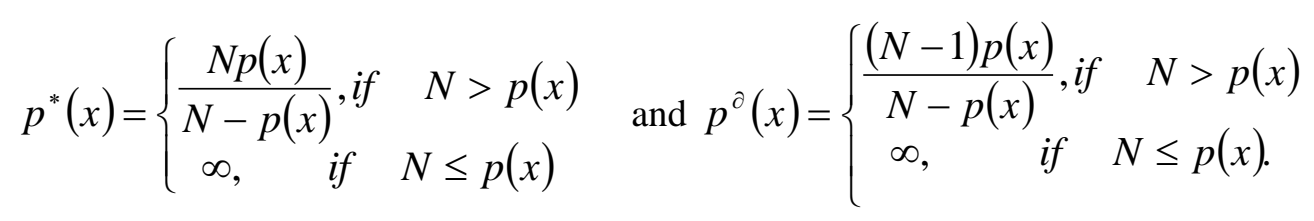

Definition 2.7 [23] Let $X$ be Banach spaces and the function $I \in C^{1}(X, R)$. We say that I satisfies Palais-Smale condition (PS) in $X$ if any sequence $\left\{u_{n}\right\}$ in $X$ such that $I\left(u_{n}\right)$ is bounded and $I^{\prime}\left(u_{n}\right) \rightarrow 0$ in $X^{*}$ as $n \rightarrow \infty$ has a convergent subsequence.

Lemma 2.8 8 (Mountain Pass Theorem) [23] Let $X$ be a Banach space and the function $I \in C^{1}(X, R)$ satisfies Palais-Smale condition. Assume that $I(0)=0$ and

(i) There exist two positive real numbers $\eta$ and $r$ such that $I(u) \geq r$ with $\|u\|=r$,

(ii) There exists $u_{1} \in X$ such that $\left\|u_{1}\right\|>r$, and $I(u)<0$.

Put $G=\left\{\varphi \in C([0,1], X): \varphi(0)=0, \varphi(1)=u_{1}\right\} . \quad$ Set $\beta=\inf \{\max \varphi([0,1]): \varphi \in G\}$. Then $\beta \geq r$ and $\beta$ is a critical value of $I$.

Throughout this paper, the following hypotheses are assumed.

(f1) $f: \Omega x R \rightarrow R$ is Carathéodory condition such that

$$
|f(x, t)| \leq c_{1}+c_{2}|t|^{\alpha(x)-1} \quad, \quad \forall(x, t) \in \Omega x R,
$$

where $c_{1}, c_{2}>0, \alpha(x) \in C_{+}(\bar{\Omega})$ and $\alpha(x)<p^{*}(x)$, 
(f2) $f(x, t)=o\left(|t|^{p^{+}-1}\right), t \rightarrow 0$; for all $x \in \Omega$,

(AR): Ambrosetti-Rabinowitz's condition; there exist $t^{*}>0$ and $\theta>p^{+}$such that $0<\theta F(x, t) \leq f(x, t) t,|t| \geq t^{*}$, for all $x \in \Omega$

where $F(x, t)=\int_{0}^{t} f(x, s) d s$ and $q^{-} \leq q(x)<p^{\partial}(x)$ for all $q(x) \in C(\partial \Omega)$.

Theorem 2.9. Assume that conditions (f1), (f2), (AR), $q^{-}>\theta, \alpha^{-}, p^{+}$and $\alpha^{-}>p^{+}$are satisfied, then problem $(P)$ has at least one nontrivial weak solution.

\section{Main Results}

Let $X$ denote the variable exponent Sobolev space $W^{1, p(x)}(\Omega)$. We say that $u \in X /\{0\}$ is a weak solution of $(\mathbf{P})$ if

$$
\int_{\Omega} a(x)|\nabla u|^{p(x)-2} \nabla u \nabla v d x+\int_{\Omega}|u|^{p(x)-2} u v d x-\int_{\partial \Omega}|u|^{q(x)-2} u v d \sigma-\int_{\Omega} f(x, u) v d x=0
$$

for all $v \in X$.

The energy functional corresponding to the problem $(P)$ is defined as $I=X \rightarrow R$

$$
I(u)=\int_{\Omega} \frac{a(x)|\nabla u|^{p(x)}+|u|^{p(x)}}{p(x)}-\int_{\partial \Omega} \frac{|u|^{q(x)}}{q(x)} d \sigma-\int_{\Omega} F(x, u) d x
$$

where $F(x, u)=\int_{0}^{u} f(x, s) d s$ and $d \sigma$ is the measure on the boundary.

Proposition 3.1 [24] If one denotes

$$
J(u)=\int_{\Omega} \frac{a(x)|\nabla u|^{p(x)}+|u|^{p(x)}}{p(x)} d x, \forall x \in X .
$$

Then $J \in C^{1}(X, R)$ and the derivative operator of $J$, denoted by $J^{\prime}$, is

$$
\left\langle J^{\prime}(u), v\right\rangle=\int_{\Omega} a(x)|\nabla u|^{p(x)-2} \nabla u \nabla v d x+\int_{\Omega}|u|^{p(x)-2} u v d x, \quad \forall u, v \in X,
$$

and one has:

(i) $\quad J^{\prime}: X \rightarrow X^{*}$ is a continuous, bounded, and strictly monotone operator,

(ii) $J^{\prime}$ is a mapping of $\left(S^{+}\right)$type, that is, if $u_{n} \rightarrow u$ (weak convergent) in $X$ and $\lim \sup _{n \rightarrow \infty}\left\langle J^{\prime}\left(u_{n}\right)-J^{\prime}(u), u_{n}-u\right\rangle \leq 0$, then $u_{n} \rightarrow u$ (strongly convergent) in $X$,

(iii) $\quad J^{\prime}: X \rightarrow X^{*}$ is a homeomorphism.

Proposition 3.2 [5,24] If one denotes

$$
\varphi(u)=\int_{\partial \Omega} \frac{|u|^{q(x)}}{q(x)} d \sigma, \forall u \in X,
$$

where $\mathrm{q}(\mathrm{x}) \in \mathrm{C}_{+}(\partial \Omega)$ and $\mathrm{q}(\mathrm{x})<p *(x)$ for any $\mathrm{x} \in \partial \Omega$, then $\varphi \in C^{1}(X, R)$ and the derivative operator of $\varphi$, denoted by $\varphi^{\prime}$, is 


$$
\left\langle\varphi^{\prime}(u), v\right\rangle=\int_{\partial \Omega}|u|^{q(x)-2} u v d \sigma, \quad \forall u, v \in X,
$$

and one has $\varphi: X \rightarrow R$ and $\varphi^{\prime}: X \rightarrow X^{*}$ are sequentially weak-strongly continuous, bounded, namely, $u_{n} \rightarrow u$ (weakly continuous) implies $\varphi^{\prime}\left(u_{n}\right) \rightarrow \varphi^{\prime}(u)$ (strongly continuous).

Therefore, from the assumption (f1), Proposition 2.6, Proposition 3.1 and Proposition 3.2, it is easy to see that $I(u) \in C^{1}(X, R)$ and the critical points $I$ are weak solutions of $(P)$. Moreover, the derivate of $I$ is the mapping $I^{\prime}: X \rightarrow X^{*}$

$$
\left\langle I^{\prime}(u), v\right\rangle=\int_{\Omega}\left(a(x)|\nabla u|^{p(x)-2} \nabla u \nabla v+|u|^{p(x)-2} u v\right) d x-\int_{\partial \Omega}|u|^{p(x)-2} u v d \sigma-\int_{\Omega} f(x, u) v d x
$$

for any $u, v \in X[6]$.

Lemma 3.3 Suppose that (f1), (f2), (AR) and $q^{-}>\theta$ are satisfied, then I satisfies the $(\boldsymbol{P S})$ condition.

Proof. Assume that $\left\{u_{n}\right\} \subset X$ is a sequence which satisfies the properties:

$$
I\left(u_{n}\right) \rightarrow C \text { and } I^{\prime}\left(u_{n}\right) \rightarrow 0 \text { in } X^{*} \text { as } n \rightarrow \infty,
$$

where $X^{*}$ is dual space of $\mathrm{X}$ and $C$ is a positive constant. We prove that $\left\{u_{n}\right\}$ possesses a convergent subsequence. First, we show that $\left\{u_{n}\right\}$ is bounded in $X$. We assume by contradiction $\left\|u_{n}\right\| \rightarrow \infty$ as $n \rightarrow \infty$. Using $(\boldsymbol{A R}) q^{-}>\theta$, (3.1), Proposition 2.2, Proposition 2.3 and considering $\left\|u_{n}\right\|>1$, for $\mathrm{n}$ large enough, we can write

$$
\begin{aligned}
& C+\left\|u_{n}\right\| \geq I\left(u_{n}\right)-\frac{1}{\theta}\left\langle I^{\prime}\left(u_{n}\right), u_{n}\right\rangle \\
& =\int_{\Omega} \frac{1}{p(x)}\left(a(x)\left|\nabla u_{n}\right|^{p(x)}+\left|u_{n}\right|^{p(x)}\right) d x-\int_{\partial \Omega} \frac{1}{q(x)}\left|u_{n}\right|^{q(x)} d \sigma-\int_{\Omega} F\left(x, u_{n}\right) d x \\
& -\frac{1}{\theta}\left(\int_{\Omega}\left(a(x)\left|\nabla u_{n}\right|^{p(x)}+\left|u_{n}\right|^{p(x)}\right) d x-\int_{\partial \Omega}\left|u_{n}\right|^{q(x)} d \sigma-\int_{\Omega} f\left(x, u_{n}\right) u_{u} d x\right) \\
& \geq\left(\frac{\varepsilon_{1}}{p^{+}}-\frac{1}{\theta}\right)|| u_{n} \|^{p^{-}}
\end{aligned}
$$

where $c_{3}>0$ is constant. Since $\theta>p^{+}$we obtain that $\left\{u_{n}\right\}$ is bounded in X. Next, we show the strong converges to $u_{n} X$ in. Since it $\left\{u_{n}\right\}$ is bounded in $X$, there exists $u$ in $X$ such that, up to a subsequence, $u_{n}$ converges weakly to $u$ in $X$. Taking into account (3.1), we have $\left\langle I^{\prime}\left(u_{u}\right), u_{n}-u\right\rangle \rightarrow 0$. So, we have

$$
\begin{aligned}
& \left\langle I^{\prime}\left(u_{n}\right), u_{n}-u\right\rangle \\
& =\int_{\Omega}\left(a(x)\left|\nabla u_{n}\right|^{p(x)-2} \nabla u_{n}\left(\nabla u_{n}-\nabla u\right)+\left|u_{n}\right|^{p(x)-2} u_{n}\left(u_{n}-u\right)\right) d x \\
& -\int_{\partial \Omega}\left|u_{n}\right|^{q(x)-2} u_{n}\left(u_{n}-u\right) d \sigma-\int_{\Omega} f\left(x, u_{n}\right)\left(u_{n}-u\right) d x .
\end{aligned}
$$

Using (f1) and Proposition 2.1, it follows 


$$
\begin{aligned}
& \left|\int_{\Omega} f\left(x, u_{n}\right)\left(u_{n}-u\right) d x\right| \leq\left|\int_{\Omega}\left(c_{1}+c_{2}\left|u_{n}\right|^{\alpha(x)-1}\right)\left(u_{n}-u\right) d x\right| \\
& \leq c_{1} \int_{\Omega}\left|u_{n}-u\right| d x+\left.\left.c_{4}|| u_{n}\right|^{\alpha(x)-1}\right|_{\alpha^{\prime}(x)}\left|u_{n}-u\right|_{\alpha(x)}
\end{aligned}
$$

where $c_{4}>0$ is constant. Because $\alpha(x)<p^{*}(x)$ (Proposition 2.6 (ii)), there exists $u$ such that $u_{n}$ converges weakly to $u$ in $X$. Thanks to the compact embedding $X \rightarrow L^{\alpha(x)}(\Omega)$, we get

$$
\begin{gathered}
u_{n} \rightarrow u(\text { strongly }) \text { in } L^{\alpha(x)} \\
u_{n} \rightarrow \text { ua.e. } x \in \Omega
\end{gathered}
$$

So,

$$
\int_{\Omega} f\left(x, u_{n}\right)\left(u_{n}-u\right) d x \rightarrow 0 .
$$

Similarly, by Proposition 2.1, Proposition 2.6 (iii) and Proposition 3.2, we have

$$
\int_{\partial \Omega}\left|u_{n}\right|^{q(x)-2} u_{n}\left(u_{n}-u\right) d \sigma \rightarrow 0 .
$$

Thus,

$$
\int_{\Omega}\left(a(x)\left|\nabla u_{n}\right|^{p(x)-2} \nabla u_{n}\left(\nabla u_{n}-\nabla u\right)+\left|u_{n}\right|^{p(x)-2} u_{n}\left(u_{n}-u\right)\right) d x \rightarrow 0 .
$$

Finally, from Proposition 3.1, we deduce that $u_{n}$ converges strongly to $u X$ in. Therefore, $I$ it satisfies the (PS) condition.

Lemma 3.4 Assume that conditions (f1), $(\boldsymbol{f} 2), q^{-}>p^{+}, \alpha^{-}$and $\alpha^{-}>p^{+}$are fulfilled. Then, there exist two positive real numbers $\rho$ and $r$ such that $I(u) \geq r$ with $\|u\|=\rho$.

Proof: For $\|u\|<1$, by Proposition 2.5, we have

$$
I(u) \geq \frac{\varepsilon_{3}}{p^{+}}\|u\|^{p^{+}}-\frac{1}{q^{-}} \int_{\partial \Omega}|u|^{q(x)} d \sigma-\int_{\Omega} F(x, t) d x
$$

where $c_{7}$ is constant. Since we have the continuous embeddings $X \rightarrow L^{q(x)}(\partial \Omega) X \rightarrow L^{p^{+}}(\Omega)$, and $X \rightarrow L^{\alpha(x)}(\Omega)$ (Proposition 2.6), there exist $c_{5}, c_{6}$ and $c_{7}$ positive constants such that for all $u \in X$

$$
|u|_{q(x), \partial \Omega} \leq c_{5}\|u\|, \quad|u|^{p^{+}} \leq c_{6}\|u\| \text { and }|u|_{\alpha(x), \Omega} \leq c_{8}\|u\| .
$$

Choose $\varepsilon>0$ small enough such that $\left(\varepsilon c_{6}^{p^{+}}+c_{\varepsilon} c_{7}^{\alpha^{-}}\right)<\frac{\varepsilon_{4}}{2 p^{+}}$. Using (f1) and (f2), we have

$$
|F(x, t)| \leq \varepsilon|t|^{p^{+}}+c_{\varepsilon}|t|^{\alpha(x)} \text {, for all }(x, t) \in \Omega \times \mathrm{R} \text {. }
$$

Thus, using (3.2) and (3.3) for $\|u\|<1$, we get

$$
\begin{aligned}
& I(u) \geq \frac{\varepsilon_{4}}{p^{+}}\|u\|^{p^{+}}-\frac{c_{5}}{q^{-}}\|u\|^{q^{-}}-\varepsilon c_{6}^{p^{+}}\|u\|^{p^{+}}-c_{\varepsilon} c_{7}^{\alpha^{-}}\|u\|^{\alpha^{-}} \\
& \geq \frac{\varepsilon_{4}}{p^{+}}\|u\|^{p^{+}}-\frac{c_{5}}{q^{-}}\|u\|^{q^{-}}-\varepsilon c_{6}^{p^{+}}\|u\|^{p^{+}}-c_{\varepsilon} c_{7}^{\alpha^{-}}\|u\|^{p^{+}} \\
& \geq \frac{\varepsilon_{4}}{2 p^{+}}\|u\|^{p^{+}}-\frac{c_{5}}{q^{-}}\|u\|^{q^{-}}
\end{aligned}
$$


Choose $c_{5} \leq \frac{q^{-} \varepsilon_{4}}{2 p^{+}}$. It follows that there exist $r>0$ s and $\rho>0$ such that $I(u) \geq r$ with $\|u\|=\rho$. The proof of Lemma 3.4 is completed.

Lemma 3.5 If (f1), (f2) and (AR) hold, there exists $\phi \in X$ such that $\|\phi\|>\eta$ and $I(t \phi)<0$ for $t>0$. Proof. Thanks to (AR), we obtain $|F(x, t)| \geq c_{8}|t|^{\theta}$ for all $(x, t) \in \Omega \times R$. Moreover, when $t>1$ is large enough, from Proposition 2.2, we obtain that

$$
\begin{aligned}
& I(t \phi)=\int_{\Omega} \frac{a(x)|\nabla t \phi|^{p(x)}+|t \phi|^{p(x)}}{p(x)} d x-\int_{\partial \Omega} \frac{|t \phi|^{q(x)}}{q(x)} d \sigma-\int_{\Omega} F(x, t \phi) d x \\
& \leq \frac{t^{p^{+}}}{p^{-}} \int_{\Omega}\left(a(x)|\nabla \phi|^{p(x)}+|\phi|^{p(x)}\right) d x-\frac{t^{q^{-}}}{q^{+}} \int_{\partial \Omega}|\phi|^{q(x)} d \sigma-c_{8} t^{\theta} \int_{\Omega}|\phi| d x
\end{aligned}
$$

Since $\theta>p^{+}$we conclude that $I(t \phi) \rightarrow-\infty t \rightarrow \infty$ as. The proof is completed.

Proof of Theorem 2.9. From Lemma 3.3, Lemma 3.4, Lemma 3.5 and $I(0)=0$, I satisfies all statements of Lemma 2.8. Therefore, $I$ has at least one nontrivial critical point, i.e., problem $(\boldsymbol{P})$ has a nontrivial weak solution. The proof is completed.

\section{Acknowledgment}

The author would like to thank anonymous referees for reading the original manuscript carefully and their valuable suggestions.

\section{References}

[1] Acerbi E. and Mingione G., "Regulary results for a class of functionals with nonstandard growth", Arch. For Rational Mech. Anal., 156, 121-140, Zbl0984.49020, 2015

[2] Allaoui M., El Amrouss A.R., Ourraoui A., "Existence and multiplicity of solutions for a Steklov problem involving the p(x)-Laplace operator", Electron. J. Differential Equations, 132, 1-12, 2012.

[3] Antontsev S. N. and Shmarev S. I., "A model porous medium equation with variable exponent of nonlinearity: existence, uniqueness and localization properties of solutions", Nonlinear Anal., 60, $515-545,2005$.

[4] Avci M. and Pankov A., "Nontrivial solutions of discrete nonlinear equations with variable exponent", J. Math. Anal. Appl., 431, 22-33,2015

[5] Ayoujil A., "On the superlinear Steklov problem involving the $\mathrm{p}(\mathrm{x})$-Laplacian", EJQTDE, 38, 1$13,2014$.

[6] Ben Ali K., "Existence results for Steklov problem involving the p(x)-Laplacian", Complex Var. and Elliptic Equ., 63, 1675-1686, 2017.

[7] Chen Y.M. and Levine S., Rao M., "Variable exponent, linear growth functionals in image restoration", SIAM J. Appl. Math., 66, 1383-1406, 2006.

[8] Diening L., "Theoretical and numerical results for electrorheological fluids", Ph.D. thesis, University of frieburg, Germany, 2002. Zbl 1022.76001 
[9] Deng SG. "Eigenvalues of the $p(x)$-Laplacian Steklov problem", J. Math Anal Appl., 339, 925937, 2008.

[10] Edmunds D, Rakosnik J., "Sobolev embeddings with variable exponent". Studia Math., 143, 267-293, 2000.

[11] Ekincioğlu I and Ayazoğlu R, "On Steklov Boundary Value Problems for p(x)Laplacian equations", Electronic Journal of Math. Analy. And Appl. (EJMAA), 5(2), 289-297, 2017.

[12] Halsey T. C., "Electrorheological fluids”, Science, 258, 761-766, 1992.

[13] Hsini M, Irzi N and Kefir K., "Nonhomogeneous p(x)-Laplacian Steklov problem with weights", Complex Variables and Elliptic Equations, https://doi.org/10.1080/17476933.2019.1597070.

[14] Fan XL, Zhang QH. "Existence of solutions for $p(x)$-Laplacian Dirichlet problem". Nonlinear Anal., 52, 1843-1852, 2003.

[15] X. Fan, Q. Zhang, and D. Zhao, "Eigenvalues of p(x)-Laplacian Dirichlet problem", J. Math. Anal. Appl., 302, 306-317, 2015.

[16] Kovăčik O., and Răkosnik J., "On spaces $L^{p(x)}(\Omega)$ and $W^{k, p(x)}(\Omega)$ ”, Czechoslovak Math. J., 41(116), 592-618, 1991.

[17] Mavinga N, Nkashama MN., "Steklov spectrum and nonresonance for elliptic equations with nonlinear boundary conditions”, Electron J Differ Equ Conf., 19, 197-205, 2010.

[18] Mashiyev R. A, Çekiç B, Avci M. and Yücedag Z., "Existence and multiplicity of weak solutions for nonuniformly elliptic equations with nonstandard growth condition", Complex Variables and Elliptic Equations, .57(5), 579-595, 2012.

[19] Mihăilescu M. and Rădulescu V., "A multiplicity result for a nonlinear degenerate problem arising in the theory of electrorheological fluids", Proceedings of the Royal Society A., 462, 2625$2641,2006$.

[20] Ruzicka M., "Electrorheological fluids: modelling and mathematical theory. Lecture notes in mathematics", Vol.1784. Berlin: Springer-Verlag; 2000.

[21] Yücedag, Z., "Existence of Solutions for $\mathrm{p}(\mathrm{x})$ Laplacian Equations Without AmbrosettiRabinowitz Type Condition”, Bull. Malaysian Math. Sci. Soc. (2) 38(3), 1023-1033, 2015.

[22] Yücedag Z., "Solutions of nonlinear problems involving p(x)-Laplacian operator", $A d v$. Nonlinear Anal., 4, 285-293, 2015.

[23] Willem M., "Minimax Theorems”, Birkhauser, Basel, 1996.

[24] Wei Z. and Chen Z., "Existence results for the $p(x)$-Laplacian with nonlinear boundary condition", Applied Math., Article ID 727398, doi:10.5402/2012/727398, 2012.

[25] Zhikov VV., "Averaging of functionals of the calculus of variations and elasticity theory", Math. USSR. Izv., 29, 33-66, 1987. 